\title{
Die EU und ihre Alkoholpolitik - Relevanz für die Bekämpfung von häuslicher Gewalt?
}

\author{
Bericht über den Kongress Building Capacity vom 24. bis 26. September 2007 \\ in Bled, Slowenien
}

Unter der Leitung des slowenischen Gesundheitsministeriums in Vorbereitung der Europaratspräsidentschaft von Slowenien in 2008 trafen sich Kommissionsmitglieder der EU, Vertreter aller EUStaaten sowie aus Nachbarländern mit dem Ziel der Weiterentwicklung und Implementierung der europäischen Alkoholpolitik sowie der Schaffung funktionstüchtiger Netzwerke in Europa. Ziel ist dabei auch die Reduktion der alkoholbedingten Sozialkosten in der EU von etwa 125 Millionen Euro im Jahr. Durch die professionelle Zusammenarbeit in zehn work packages soll dieses Ziel auf internationaler, nationaler und regionaler Ebene in der Laufzeit des Projektes von Mai 2007 bis April 2010 erreicht werden, der dritte Fachkongress fand vom 2. bis 5. April 2008 in Barcelona statt.

Unter der Überschrift des work package 10 „Prävention von alkoholbedingten Unfällen und Verletzungen“ referierten Spezialisten über

- inequalities, accidents and injuries, and alcohol;

- violence and injury prevention;

- alcohol and suicide.

Erfreulicherweise war den Organisatoren dieses Kongresses auch der Zusammenhang zwischen häuslicher Gewalt und Alkohol bekannt, so dass ich als Vertreterin der Kommission Gewalt gegen Frauen und Kinder des djb zu der Sonderproblematik „domestic violence and alcohol“ referieren durfte. Im Zuge dieses Vortrages war festzustellen, dass zum einen nach wie vor eine einheitliche Definition und Verwendung des Begriffes häusliche Gewalt, besser Partnergewalt, fehlt, zum anderen aber auch die Auswirkungen von Alkohol in diesem $\mathrm{Zu}$ sammenhang bisher kaum beleuchtet werden. Insoweit kann dabei im Wesentlichen nur auf die umfassende Studie der Weltgesundheitsorganisation, the WHO multi-country study on women's health and domestic violence against women ${ }^{1}$ mit den ersten Veröffentlichungen im November 2005 zurückgegriffen werden, die im erheblichen Umfang den Zusammenhang zwischen Alkohol und Partnergewalt feststellt.

Dabei geht auch die WHO davon aus, dass Partnergewalt im deutlich höheren Prozentsatz von Männern gegenüber Frauen ausgeübt wird. Welt- weite wissenschaftliche Studien aus empirischen Erhebungen der letzten 30 Jahre, so insbesondere die im Juli 2006 veröffentlichte Studie der UN Secretary General's in depth study on all forms of violence against women ${ }^{2}$ haben die diesbezügliche These der internationalen Frauenbewegung bestätigt. Die UN-Studie benennt dabei ebenfalls regelmäßigen Alkoholkonsum als einen erheblichen Risikofaktor.

Die Kongressteilnehmerinnen und -teilnehmer waren offensichtlich über die hohe Verbreitung von häuslicher Gewalt in allen Bevölkerungsschichten, den engen Zusammenhang von Alkohol und Partnergewalt und die immensen ökonomischen Kosten, die durch Partnergewalt ausgelöst werden, überrascht und auch entsetzt.

So ergeben Schätzungen, nachzulesen in den fact sheets - Intimate partner violence and alcohol - der WHO aus $2006^{3}$, unter anderem für ausgesuchte Länder folgende Größenordnungen für die durch Partnergewalt entstehenden ökonomischen Kosten, wobei hierzu Gesundheitskosten, Kosten der Justiz, Kosten für die Änderung der Wohnsituation, Verlust von Arbeitseinkommen etc. zählen:

In den Vereinigten Staaten von Amerika wurden die Kosten auf 12,6 Milliarden US-Dollar im Jahr geschätzt (2002). Dabei hatten etwa 55 Prozent der Täter Alkohol konsumiert. Auch in England und Wales wurde festgestellt, dass die Täter in etwa 32 Prozent der Fälle unter Alkoholeinfluss standen. Die geschätzten Kosten lagen im Jahr 2004 bei 5,7 Milliarden Pfund, zusätzlich 17 Milliarden Pfund Kosten im Zusammenhang mit den Opfern. In Kanada lagen die Kosten für die medi-

1 Verfügbar unter www.who.int/violence_injury prevention/publications/violence/en/index.html.

2 Verfügbar unter www.Un/org/womenwatch/daw/ vaw/SGstudy/vaw.htm, s. Beitrag hierzu Gage-Lindner, N. in aktuelle informationen 2006, Heft 2, S. 34

3 Fact sheets 2006, Seite 5, Fußnote 1, s.a. die Auswertung von über 119 Studien in: Waters, H. et alia, The economic dimensions of interpersonal violence (2004), Zahlen zu Partnergewalt: S. 17 ff., http://www. who.int/violence_injury_prevention/publications/ violence/economic_dimensions/en/index.html und Hagemann-White $\bar{C}$ et alia (2006), Combating Violence Against Women, Stocktaking study on the measures and actions taken in Council of Europe member states, S. 8 ff., http://www.coe.int/t/e/ human_rights/equality/PDF_CDEG(2006)3_E.pdf.

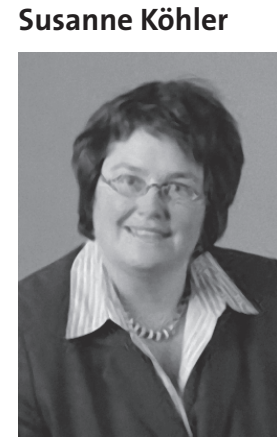

Mitglied der Kommission Gewalt gegen Frauen und Kinder des djb; Vorsitzende des Landesverbands

Sachsen und der Regionalgruppe Dresden im djb; Fachanwältin für Familienrecht und Arbeitsrecht, Dresden 
zinische Behandlung von weiblichen Opfern geschlechtsbezogener Gewalt bei geschätzten 1,1 Milliarden US-Dollar (2004).

Auch nationale Studien über häusliche Gewalt stellen häufig bei Tätern Alkoholkonsum fest, wobei die Schätzungen zwischen den einzelnen Ländern variieren. Festzustellen ist, dass auch Opfer von Partnergewalt häufig unter Alkoholeinfluss standen, dies aber in einem niedrigeren Ausmaß als die Täter. Gewalterfahrung in der Partnerschaft kann dabei das Opfer zu Alkoholmissbrauch veranlassen.

Sowohl die Studienauswertungen der WHO als auch die jüngste Studie des Generalsekretärs der UN zur Gewalt gegen Frauen machen deutlich, dass bei der Erarbeitung von Maßnahmen gegen überhöhten Alkoholkonsum bzw. alkoholbeeinflusste Partnergewalt gesellschaftliche Vorstellungen über Alkoholkonsum, Geschlechterrollen und Gewaltverhalten nicht unberücksichtigt bleiben dürfen, da diese das Risiko alkoholbedingter Partnergewalt beeinflussen. Dabei werden in manchen Gesellschaftsformen erhöhter Alkoholkonsum und gewaltsames Verhalten als männlich bewertet und sind damit positiv unterlegt bzw. werden nicht als zu missbilligen angesehen. Exzessiver Alkoholkonsum steigert meist die finanziellen Probleme, damit auch die Konflikte zwischen den Partnern, was wiederum das Risiko von Partnergewalt steigen lässt. Partnergewalt ist ernster und endet häufiger in physischen Verletzungen, wenn der Täter unter Alkoholeinfluss steht. Diese Feststellungen können wenig erstaunen, da Alkohol di- rekte Auswirkungen auf die kognitiven und physischen Körperfunktionen hat und die Selbstkontrolle reduziert.

Die WHO geht davon aus, dass durch die Reduzierung von unmäßigem Alkoholkonsum auch die Häufigkeit von Partnergewalt vermindert werden kann und empfiehlt folgende Maßnahmen:

- Reduzierung der Möglichkeiten, Alkohol zu erwerben;

- Regelungen zur Preisgestaltung von Alkohol;

- Umfassende Behandlungsmaßnahmen bei Alkoholmissbrauch;

- Früherkennung und schnelle Intervention bei Alkoholproblemen.

Dabei ist jedoch zu beachten, dass es noch keine gesicherten Erhebungen dazu gibt, dass diese Interventionen in Ländern mit hohem Pro-Kopf-Einkommen gleich effektiv sind wie in Ländern mit niedrigem und mittlerem Pro-Kopf-Einkommen.

In der auf den Vortrag folgenden Diskussion wurde deutlich, dass erheblicher Handlungsbedarf dahingehend besteht, den genauen Zusammenhang zwischen Alkoholkonsum und Partnergewalt in den einzelnen Mitgliedstaaten näher zu erfassen, um damit auch geeignete Instrumente zur Verhinderung von Partnergewalt zu entwickeln. Dabei wurde insbesondere auch die internationale Zusammenarbeit als wesentliche Voraussetzung für eine erfolgreiche Prävention und Intervention erkannt.

\section{Erkenntnisse aus Fachkonferenzen in Europa}

\section{Nancy \\ Gage-Lindner}

Sabine

Kräuter-Stockton

\author{
Sylvia \\ Cleff Le Divellec
}

Der Deutsche Juristinnenbund nutzt konsequent die Chance, auf Fachkonferenzen zum fachlichen Diskurs und zur Außenwirkung des Verbands beizutragen'. So auch die Mitglieder der Kommission Gewalt gegen Frauen und Kinder ${ }^{2}$. Im Folgenden geben wir Eindrücke aus einem Ausschnitt der Fachgespräche wieder, die wir in letzter Zeit geführt haben ${ }^{3}$.

\section{Berner Tagung für klinische Rechtsmedizin Institut für Rechtsmedizin der Universität Bern 31. August bis 1. September 2008 in Bern}

Dank der traditionellen Schnittstelle der Rechtsmedizin zwischen der Humanmedizin und der Justiz ist eine Kooperation des djb mit dieser Fachrichtung naheliegend und so verfolgt die Kommission Gewalt gegen Frauen und Kinder seit mehreren Jahren extensiv dieses Potential ${ }^{4}$. Während allerdings die Ursprünge der Rechtsmedizin im öffentlichen Gesundheitswesen verortet sind, d.h. dem gesamtgesellschaftlichen Interesse am Erhalt der Gesundheit dienen, zeichnet sich dieses Fach seit Jahrzehnten schon rechtssystematisch durch eine Ferne zur klinischen Medizin aus, d.h. zur gesundheitlichen Versorgung per se. So kommt es

1 Vgl. hierzu die Rubrik „djb präsent“ in diesem und jedem Heft.

2 Wir danken unseren Kolleginnen Sylvia Cleff der Kommission Öffentliches Recht, Europa- und Völkerrecht und Christiane Schreiber der Kommission Ältere Menschen für ihre Beiträge.

3 Siehe auch den Beitrag von Susanne Köhler in diesem Heft.

4 Zur ersten gemeinsamen Veranstaltung kam es 2003, worüber in den aktuellen informationen 2003 berichtet wurde. 